\title{
Output Feedback Trajectory Tracking Control of a Car-like Drive Wheeled Mobile Robot Using RBF Neural Network
}

\author{
Yasamin Raeisi, Khoshnam Shojaei, Abbas Chatraei \\ Department of Electrical Engineering, Najaf Abad Branch, Islamic Azad University, Esfahan, Iran \\ e-mail: yasamin_raisi@yahoo.com, khoshnam.shojaee@gmail.com, abbas.chatraei@gmail.com
}

\begin{abstract}
This paper addresses the output feedback trajectory tracking control problem of Ackerman steering-drive wheeled mobile robots under nonholonomic constraints in the presence of model uncertainties without velocity measurement. A RBF neural network and a linear observer are employed to construct the controller for constrained robot with only position measurement. The proposed controllers employ saturation-type adaptive-neural control laws to effectively compensate for the uncertain parameters, unmodeled dynamics and unknown bounded disturbances. Lyapunov-based stability analyses are utilized to guarantee that tracking errors are uniformly ultimately bounded and exponentially converge to a small ball containing the origin. The simulation results are presented to illustrate the tracking effectiveness of the controller.
\end{abstract}

Keywords-Adaptive-neural control; RBF NN; Nonholonomic WMR; Trajectory tracking;

\section{INTRODUCTION}

Applications for wheeled mobile robots (WMRs) are found in many areas like military operations, surveillance, entertainment, transportation, planetary exploration and mining [1]. An important class of nonholonomic mechanical systems is wheeled mobile robots. Design of tracking and stabilizing controllers for these systems are challenging because of well-known Brockett's theorem [2]. Among various motion control problems of nonholonomic WMRs, most of researches have been concentrated on the tracking of a geometric path with an associated timing law so-called trajectory tracking [3].

After seminal works in the references [4]-[8], many works have been published to introduce trajectory tracking controllers for WMRs. Initially researchers designed their motion controllers based on the kinematic model [3]-[10] and later progressed towards tracking controllers using the dynamic model. The determination of accurate kinematic and dynamic models is always a difficult thing to do and the uncertainty in the model cannot be avoided, many researchers proposed adaptive and robust controllers to solve the trajectory tracking problem of WMRs. For example, see [11]-[16].

The output feedback control problem is of practical importance since most commercial WMRs are not equipped with the velocity sensors. The design of output feedback controller is a challenging task because of the presence of parametric and nonparametric uncertainties in the system model as well as the existence of nonholonomic constraints of WMRs. In addition, the separation principle does not hold for the nonlinear system. Both the trajectory tracking and the path following problems have their own complexity as the carlike mobile robot kinematic model is nonholonomic. In [17] a simple and effective fuzzy PID controller for kinematics model of a car-like mobile robot is presented. The proposed controller can solve the path following problem for a car-like mobile robot when the initial state is an arbitrary point. Also, Luca et al. designed a trajectory tracking controller as well as comparing several feedback solutions for point stabilization [18]. Some researchers have designed controllers using the kinematic model with wheel skidding and slipping [19]-[20].

However, it is well known that for high enough velocity or slippery enough surface the car motion cannot be described accurately by kinematic models and one should use dynamical model to design accurate trajectory tracking algorithms. Some papers using the virtual vehicle approach proposed the carlike robot controller by the dynamic model. The controller uses the idea of virtual car with feedback on the path, so that the real car follows the virtual car [21]-[22].

The main contribution of this paper is to design an adaptive trajectory tracking output feedback controller using RBF neural network in presence of uncertainties in the model of nonholonomic Ackerman steering-drive wheeled mobile robots where radial basis function (RBF) neural network (NN) is used to compensate the nonlinearities of model robot. In this paper, an adaptive output feedback trajectory tracking controller is designed based on the reference [23] which is presented in the motion control of robot manipulators. In contrast to many previously proposed controllers, our proposed controllers can easily be applied to all types of the WMRs by choosing a suitable set of output equations and our proposed method can compensate for both parametric and non-parametric uncertainties such as friction and external disturbances and it does not require any transformation matrix in the design of the output feedback controller.

The rest of the paper is organized as follows. Section 2 reviews the kinematic and dynamic formulation of nonholonomic WMRs. A WMR models is introduced in this section which is necessary for the development of the controllers. Section 3 proposes an output feedback trajectory tracking controller. Simulation results are separately presented in order to evaluate the performance of the controllers in section 4. Finally, the paper is concluded in section 5 .

\section{PROBLEM FORMULATION}

\section{A. Kinematic and Dynamic models of WMRs}

Consider a class of nonholonomic robotic systems subject to $m$ constraints in the following form [6]:

$$
\begin{aligned}
& M(q) \ddot{q}+C(q, \dot{q}) \dot{q}+\tau_{d}(t, \dot{q})=B(q) \tau-A^{T}(q) \lambda \\
& \begin{array}{l}
A(q) \dot{q}=0 \\
\quad q=\left[q_{1}, q_{2}, \ldots, q_{n}\right]^{T}: \text { vector of generalized coordinates, }
\end{array}
\end{aligned}
$$


$\tau \in \mathfrak{R}^{(n-m) \times 1}:$ vector of actuators inputs,

$M(q) \in \mathfrak{R}^{n \times n}$ :symmetric positive-definite inertia matrix,

$C(q, \dot{q}) \in \mathfrak{R}^{n \times n}:$ centripetal and Coriolis matrix,

$\tau_{d}(t, \dot{q}) \in \Re^{n \times 1}:$ bounded unstructured uncertainties,

$B(q) \in \mathfrak{R}^{n \times(n-m)}$ : input transformation matrix,

$A(q) \in \mathfrak{R}^{m \times n}:$ full-rank matrix,

$\lambda \in \mathfrak{R}^{m \times 1}$ : vector of Lagrange multipliers,

$S(q)=\left[s_{1}(q), s_{2}(q), \ldots, s_{n-m}(q)\right]^{T}$ : full-rank matrix that is made up of a set of smooth and linearly independent vector fields, $s_{i}(q) \in \mathfrak{R}^{n}, i=1, \ldots, n-m$, in the null space of $A(q)$ i.e. $A(q) S(q)=0$.

Considering (2), one may find a vector of pseudovelocities of the system as $v(t)=\left[v_{1}(t), v_{2}(t), \ldots, v_{n-m}(t)\right]^{T}$ such that

$\dot{q}=S(q) v(t)=s_{1}(q) v_{1}+\ldots+s_{n-m}(q) v_{n-m}$

Differentiating (3) yields $\ddot{q}=\dot{S}(q) v+S(q) \dot{v}$ which is substituted in (1) and the result is multiplied by $S^{T}(q)$ to give the following dynamic equation:

$M_{1}(q) \dot{v}(t)+C_{1}(q, \dot{q}) v(t)+\tau_{d 1}(t, q, \dot{q})=B_{1}(q) \tau$

$M_{1}(q)=S^{T}(q) M(q) S(q)$,

$C_{1}(q, \dot{q})=S^{T}(q) M(q) \dot{S}(q)+S^{T}(q) C(q, \dot{q}) S(q)$,

$B_{1}(q)=S^{T}(q) B(q)$,

$\tau_{d 1}(t, q, \dot{q})=S^{T}(q) \tau_{d}(t, \dot{q})$

To consider the actuator dynamics, it is assumed that the robot is actuated by $n-m$ similar brushed DC motors with mechanical gears. According to Fig. 1, the electrical equation of each motor armature is written as follows:

$u_{a}=L_{a} \frac{d i_{a}}{d t}+R_{a} i_{a}+k_{b} \dot{\theta}_{m}$

where $k_{b}$ is the back EMF constant, $R_{a}$ and $L_{a}$ denote the resistance and inductance of the motor armature and $u_{a}$ is the voltage input. By ignoring the inductance of the armature circuit, and considering the relation between torque and armature current (i.e. $\tau_{m}=k_{\tau} i_{a}$ ), the delivered torque to the WMR system by actuators is given by $\tau=k_{1} u_{a}-k_{2} \dot{\theta}$ which may be re-written as [24]:

$\tau=k_{1} u_{a}-k_{2} X_{1} v$,

where $k_{1}=\left(n k_{\tau} / R_{a}\right), k_{2}=n k_{b} k_{1}, n$ is gear ratio, $k_{\tau}$ is torque constant and $X_{1} \in \mathfrak{R}^{(n-m) \times(n-m)}$ is a transformation matrix which transforms wheels velocities to pseudovelocities vector. After substituting (7) in (4), one obtains:

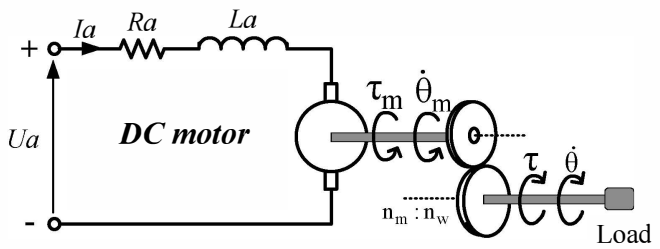

Fig. 1. Drive system for the actuation of the non-holonomic WMR.

$$
\begin{gathered}
M_{1}(q) \dot{v}(t)+C_{1}(q, \dot{q}) v(t)+k_{2} B_{1}(q) X_{1}(q) v(t) \\
+\tau_{d 1}(t, q, \dot{q})=k_{1} B_{1}(q) u_{a}
\end{gathered}
$$

Definition 1: Given a smooth bounded desired trajectory $y_{d}(t)=h\left(q_{d}(t)\right):[0, \infty) \rightarrow \mathfrak{R}^{n}$ which is generated by a reference nonholonomic WMR, and assuming that $q_{d}$ satisfies the velocity constraints (2), then the kinematic and dynamic tracking control problem discussed in this paper is to design a feedback control law for the system (3) and (8) with output equation $y(t)=h(q(t))$ such that the output tracking errors, $e(t):=y(t)-y_{\alpha}(t)$, are UUB in the presence parametric and nonparametric uncertainties in the system model when velocity measurements are not available for feedback.

Following assumptions are essential for the development of the control algorithms in the next sections:

Assumption 1: The desired trajectory $y_{d}(t)$ is chosen such that $y_{d}(t), \dot{y}_{d}(t)$ and $\ddot{y}_{d}(t)$ are all bounded signals.

Assumption 2: Since the kinematic parameters of the nonholonomic WMRs are geometric and easy to measure, such parameters are reasonably considered to be certain.

\section{B. Reduced model of the nonholonomic WMR}

A new representation of the nonholonomic WMRs models which are given by (3) and (8) is developed here to help us utilize the classic control algorithms of robot manipulators. The idea is borrowed from the Cartesian control of robotic manipulators [25] by choosing a suitable set of output equations as follows:

$$
y=h(q)=\left[h_{1}(q), h_{2}(q), \ldots, h_{n-m}(q)\right]^{T}
$$

where $y \in \mathfrak{R}^{n-m}$ is new position variable. By differentiating output equation(9) and substituting(3), one gets

$\dot{y}=J(q) v$,

where $J(q):=J_{h}(q) S(q) \in \Re^{(n-m) \times(n-m)}$ and $J_{h}(q)=\partial h / \partial q$. In fact, the output equations (9) must be chosen such that $J(q)$ is invertible. Differentiating (10) again yields $\ddot{y}=\dot{J}(q) v+J(q) \dot{v}$ which may be re-written as follows:

$$
\dot{v}=J^{-1}(q) \ddot{y}-J^{-1}(q) \dot{J}(q) v
$$

Substituting (10), (11) into (8) yields: $M_{1} J^{-1} \ddot{y}+\left(C_{1}-M_{1} J^{-1} \dot{J}\right) J^{-1} \dot{y}+D_{2} \operatorname{sgn}(\dot{q})+d$ $+\left(k_{2} B_{1} X_{1}+D_{1}\right) J^{-1} \dot{y}=k_{1} B_{1} u_{a}$

where the arguments have been dropped for the notation simplicity. Multiplying both sides of (12) by $J^{-T}$ yields the following input-output model: 


$$
M_{2}(q) \ddot{y}+C_{2}(q, \dot{y}) \dot{y}+X_{2}(q) \dot{y}+\tau_{d 2}(t, q, \dot{y})=B_{2}(q) u_{a}
$$

where

$$
\begin{aligned}
& M_{2}(q)=k_{1}^{-1} J^{-T}(q) M_{1}(q) J^{-1}(q), \\
& C_{2}(q, \dot{y})=k_{1}^{-1} J^{-T}(q)\left(C_{1}\left(q, S(q) J^{-1}(q) \dot{y}\right)-M_{1} J^{-1}(q) \dot{J}(q)\right) J^{-1}(q), \\
& X_{2}(q)=k_{1}^{-1} J^{-T}(q)\left(k_{2} B_{1}(q) X_{1}(q)+D_{1}\right) J^{-1}(q), \\
& \tau_{\alpha 2}(t, q, \dot{y})=k_{1}^{-1} J^{-T}(q)\left(D_{2} \operatorname{sgn}\left(S(q) J^{-1}(q) \dot{y}\right)+d(t)\right), \\
& B_{2}(q)=J^{-T}(q) B_{1}(q)
\end{aligned}
$$

The input-output formulation (13) is essential for the development of the controllers in the next sections.

Remark 1: It should be noted that the resulting internal dynamics of the WMR input-output model (13) depends on the output choice in (9). The effectiveness of the tracking controller design based on (13) hinges upon the stability of such internal dynamics. Yun and Yamamoto [26] investigated the stability properties of the internal dynamics of a nonholonomic WMR for the case of look-ahead control method. The look-ahead control takes the coordinates of a reference point in the front of a mobile robot as the output equation. By designing a nonlinear controller, the reference point can follow any desired trajectory. They showed that the internal dynamics are stable when the WMR is commanded to move forward, but the internal dynamics are unstable when it moves backward. Based on the presented stability results on the internal dynamics of WMRs in [26], the look-ahead control method is adopted in this paper to choose suitable output equations. Otherwise, it is necessary to analyze the stability of the resulting internal dynamics for every new set of output equations (9) which is chosen by the designer.

Property 1: The inertia matrix $M_{2}$ is a symmetric and positive-definite matrix which is upper and lower bounded by $\bar{m}_{1}\|x\|^{2} \leq x^{T} M_{2}(q) x \leq \bar{m}_{2}\|x\|^{2}$, where $\bar{m}_{1}$ and $\quad \bar{m}_{2}$ are positive scalar constants.

Property 2: The matrix $\dot{M}_{2}-2 C_{2}$ is skew symmetric, i.e.

$$
x^{T}\left(\dot{M}_{2}-2 C_{2}\right) x=0 \quad \forall x \in \Re^{n}
$$

Property 3: The centripetal-Coriolis matrix satisfies the following relationship

$$
C_{2}\left(q, x_{1}\right) x_{2}=C_{2}\left(q, x_{2}\right) x_{1} \quad \forall x_{1}, x_{2} \in \Re^{n}
$$

\section{DESIGN OF THE STATE FEEDBACK TRACKING CONTROLLER}

\section{A. RBF Neural network}

In this proposed work an RBF neural network is used to compensate for the unknown dynamics part. RBF neural networks with fixed centers and widths provide a good choice for approximation of unknown dynamics part. The RBF NN that we use are of the general form $f(x)=W^{T} \varphi(x)+\varepsilon$. Where $\mathrm{W}$ represents the matrix of neural network weights, $\varphi(x)$ is a vector of smooth basis functions, $\varepsilon$ represents the neural network construction error.

Therefore, in this paper, we employ the RBF $\mathrm{NN}$ to approximate an unknown continuous function $f(x)$ as follows $\hat{f}(x)=\hat{W}^{T} \varphi(x)$. where $\hat{W}$ is the estimate of weight vector $\mathrm{W} . \varphi(x)$ is Gaussian type of function, that is

$$
\varphi_{j}(x)=\exp \left(-\left\|x-c_{j}\right\|^{2} / \sigma_{j}^{2}\right)
$$

Where $c_{j}$ and $\sigma_{j}$ represent the center and the spread of basis function, respectively. $\left\|x-c_{j}\right\|^{2}$ is a norm of the vector $x-c_{j}$. This neural network construction error can be made arbitrarily small if $\mathrm{N}$ is sufficiently large, i.e. $\|\varepsilon\|<\varepsilon_{N}$ for some $\varepsilon_{N}>0$ where $\mathrm{N}$ denotes the number of nodes of the network.

Assumption 3: The neural network ideal weights are all bounded signals so that $\|\mathrm{W}\|_{\mathrm{F}} \leq \mathrm{W}_{\mathrm{M}}$.

\section{B. Controller development}

In this section, an output feedback trajectory tracking controller is designed based on the theoretical results of the reference [23] on the motion control of robotic manipulators.

Assumption 4: Measurements of the output vector is available in real-time by using a localization system.

Define the observation error as $z:=y-\hat{y}$ and consider the following definitions:

$$
\begin{aligned}
& \dot{y}_{r}:=\dot{y}_{d}-\Lambda\left(\hat{y}-y_{d}\right)=\dot{y}_{d}-\Lambda e+\Lambda z \\
& s_{1}:=\dot{y}-\dot{y}_{r}=\dot{e}+\Lambda e-\Lambda z \\
& \dot{y}_{o}:=\dot{\hat{y}}-\Lambda z \\
& s_{2}:=\dot{y}-\dot{y}_{o}=\dot{z}+\Lambda z
\end{aligned}
$$

where $\Lambda \in \mathfrak{R}^{(n-m) \times(n-m)}$ denotes a diagonal positive definite gain matrix.

the following tracking controller is proposed:

$$
\mathrm{u}_{\mathrm{a}}=\mathrm{B}_{2}^{-1}(\mathrm{q})\left(-\mathrm{K}_{1}\left(\dot{\mathrm{y}}_{\mathrm{o}}-\dot{\mathrm{y}}_{\mathrm{r}}\right)-\mathrm{K}_{2}(\mathrm{e}+\mathrm{z})-\hat{W}^{T} \varphi(x)\right)
$$

where $\hat{W}$ is updated by the following update rule:

$$
\dot{\hat{W}}=\Gamma \varphi\left(s_{1}+s_{2}\right)^{T}-\Gamma \sigma\left(\hat{W}-W_{0}\right)
$$

Where $\Gamma=\gamma I$ denotes the adaptation gain, $\sigma$ is a small positive number and $W_{0}$ is a priori estimate of the parameters and in this instance $W_{0}=0$ then:

$$
\dot{\hat{W}}=\Gamma \varphi\left(s_{1}+s_{2}\right)^{T}-\Gamma \sigma \hat{W}
$$

Then, considering that $s_{1}-s_{2}=\dot{y}_{o}-\dot{y}_{r}$ and substituting (21) in (13), the closed-loop system dynamics may be achieved as:

$$
\begin{aligned}
M_{2}(q) \dot{s}_{1}= & -C_{2}(q, \dot{y}) s_{1}-\left(K_{1}+X_{2}\right) s_{1}-K_{2} e+K_{1} s_{2} \\
& -K_{2} z-g_{c}-C_{2}\left(q, \dot{y}_{r}\right) s_{1}+\xi \\
f(x)=\xi= & -M_{2}(q) \ddot{y}_{r}-C_{2}\left(q, \dot{y}_{r}\right) \dot{y}_{r}-X_{2} \dot{y}_{r}-\tau_{d 2}
\end{aligned}
$$

which $\xi$ denotes the uncertain nonlinearities including unknown WMR parameters (such as mass and moment of inertia), unknown actuators parameters, friction, unmodeled dynamics and external disturbances. 
The following linear observer [23] is utilized to estimate the velocity vector:

$\dot{\hat{y}}=\dot{\hat{y}}_{o}+\Lambda z+k_{d} z$,

$\ddot{\hat{y}}_{o}=\ddot{y}_{r}+k_{d} \Lambda z$

where $k_{d} \in \mathfrak{R}$ is the observer gain which is a positive real constant. The initial conditions for the observer are chosen as follows: $\dot{\hat{y}}_{o}(0)=-\left(\Lambda z(0)+k_{d} z(0)\right), \hat{y}(0)=y(0), z(0)=\dot{\hat{y}}(0)=0$.

The interested reader is referred to [23] for details of the observer definition and derivation. Since from (26), one can write $\ddot{\hat{y}}=\ddot{\hat{y}}_{o}+\Lambda \dot{z}+k_{d} \dot{z}$ it is straightforward to show that (26) and (27) are equivalent to $\dot{s}_{1}=\dot{s}_{2}+k_{d} s_{2}$ which is together with (24) yields the following error equation

$$
\begin{aligned}
& M_{2}(q) \dot{s}_{2}=-C_{2}(q, \dot{y}) s_{2}-\left(k_{d} M_{2}(q)-K_{1}\right) s_{2}-\left(K_{1}+X_{2}\right) s_{1} \\
& -K_{2} e-K_{2} z-g_{o}+C_{2}\left(q, \dot{y}_{r}+s_{1}\right) s_{2}-C_{2}\left(q, s_{1}\right)\left(2 \dot{y}_{r}+s_{1}\right)+\xi
\end{aligned}
$$

\section{Stability Analysis}

The stability of the proposed output feedback tracking controller is summarized by the following theorem.

Theorem 1: Consider the reduced model of the nonholonomic wheeled mobile robot which is denoted by (16). Given a bounded continuous desired trajectory under assumptions 1-4, the output feedback controller (21), (23), (26) and (27) guaranties that the tracking and observation errors are uniformly ultimately bounded and exponentially converge to small ball containing the origin.

Proof: Consider following Lyapunov function candidate:

$$
\begin{aligned}
V(t)= & \frac{1}{2} e^{T} K_{2} e+\frac{1}{2} s_{1}^{T} M_{2}(q) s_{1}+\frac{1}{2} z^{T} K_{2} z \\
& +\frac{1}{2} s_{2}^{T} M_{2}(q) s_{2}+\frac{1}{2} \operatorname{tr}\left\{\dot{\tilde{W}}^{T} \Gamma^{-1} \tilde{W}\right\}
\end{aligned}
$$

where $\tilde{W}=W-\hat{W}$ denotes the vector of parameters estimation error. By differentiation (29) along (18), (20), (24) and (28), using property 2 and the fact that $\dot{\hat{W}}=-\dot{\tilde{W}}$, we have:

$$
\begin{aligned}
& \dot{V}(t)=-z^{T} K_{2} \Lambda z-s_{1}^{T} K_{1} s_{1}-e^{T} K_{2} \Lambda e-s_{1}^{T} X_{2} s_{1}+e^{T} K_{2} \Lambda z \\
& -s_{2}^{T}\left(k_{d} M_{2}(q)-K_{1}\right) s_{2}-s_{1}^{T} K_{2} z-s_{2}^{T} K_{2} e-s_{2}^{T} X_{2} s_{1} \\
& -s_{1}^{T} C_{2}\left(q, \dot{y}_{r}\right) s_{1}+s_{2}^{T} C_{2}\left(q, \dot{y}_{r}+s_{1}\right) s_{2}-s_{2}^{T} C_{2}\left(q, 2 \dot{y}_{r}+s_{1}\right) s_{1} \\
& +\left(s_{1}+s_{2}\right)^{T}\left(\tilde{W}^{T} \varphi(x)+\varepsilon\right)-\operatorname{tr}\left\{\tilde{W}^{T} \Gamma^{-1} \dot{\hat{W}}^{T}\right\}
\end{aligned}
$$

Briefly writing, by considering the facts that

$\|K\|\left\|x_{1}\right\|\left\|x_{2}\right\| \leq \frac{1}{2}\|K\| x_{1}^{T} x_{1}+\frac{1}{2}\|K\| x_{2}^{T} x_{2}, \forall x_{1}, x_{2} \in \Re^{n}$

one may write (30) as follows:

$$
\begin{aligned}
\dot{V}(t) \leq & -\lambda_{\min }(A)\|e\|^{2}-\lambda_{\min }(B)\left\|s_{1}\right\|^{2}-\lambda_{\min }(C)\|z\|^{2} \\
& -\lambda_{\min }(D)\left\|s_{2}\right\|^{2}+\left(s_{1}+s_{2}\right)^{T} \tilde{W}^{T} \varphi(x) \\
& +\left\|\left(s_{1}+s_{2}\right)^{T}\right\|\|\varepsilon\|-\operatorname{tr}\left\{\tilde{W}^{T} \Gamma^{-1} \dot{\hat{W}}\right\}
\end{aligned}
$$

where $\lambda_{\min }($.$) is used to denote the minimum eigenvalue of$ a matrix and

$$
\begin{aligned}
& A=K_{2} \Lambda-\frac{1}{2}\left\|K_{2} \Lambda\right\| I_{n-m}-\frac{1}{2}\left\|K_{2}\right\| I_{n-m}, \\
& B=K_{1}+X_{2}-\frac{1}{2}\left\|K_{2}\right\| I_{n-m}-\frac{1}{2}\left\|X_{2}\right\| I_{n-m}-\mu_{1} I_{n-m}-\frac{1}{2} \mu_{3} I_{n-m}, \\
& C=K_{2} \Lambda-\frac{1}{2}\left\|K_{2} \Lambda\right\| I_{n-m}-\frac{1}{2}\left\|K_{2}\right\| I_{n-m}, \\
& D=k_{\alpha} M_{2}(q)-K_{1}-\frac{1}{2}\left\|K_{2}\right\| I_{n-m}-\frac{1}{2}\left\|X_{2}\right\|_{n-m}-\mu_{2} I_{n-m}-\frac{1}{2} \mu_{3} I_{n-m}
\end{aligned}
$$

Now, by substituting the adaptive law (23) in (32), one gets:

$$
\begin{gathered}
\dot{V}(t) \leq-\lambda_{\min }(A)\|e\|^{2}-\lambda_{\min }(B)\left\|s_{1}\right\|^{2}-\lambda_{\min }(C)\|z\|^{2} \\
-\lambda_{\min }(D)\left\|s_{2}\right\|^{2}+\left(s_{1}+s_{2}\right)^{T} \tilde{W}^{T} \varphi(x)+\frac{1}{2}\left\|s_{1}+s_{2}\right\|^{2} \\
+\frac{1}{2}\|\varepsilon\|^{2}-\operatorname{tr}\left\{\tilde{W}^{T} \Gamma^{-1}\left(\Gamma \varphi\left(s_{1}+s_{2}\right)^{T}-\Gamma \sigma \hat{W}\right)\right\}
\end{gathered}
$$

From (33), one may choose $\Lambda, K_{1}, K_{2}$, and $k_{d}$ such that matrices A, B, C, and D are all positive definite. By simplify experssion according to the matrix laws we have:

$$
\begin{aligned}
& \dot{V}(t) \leq-\lambda_{\text {min }}(A)\|e\|^{2}-\lambda_{\text {min }}(B)\left\|s_{1}\right\|^{2}-\lambda_{\text {min }}(C)\|z\|^{2} \\
& -\lambda_{\text {min }}(D)\left\|s_{2}\right\|^{2}+\frac{1}{2}\left\|s_{1}+s_{2}\right\|^{2}+\frac{1}{2}\|\varepsilon\|^{2}+\operatorname{tr}\left\{\tilde{W}^{T} \sigma \hat{W}\right\}
\end{aligned}
$$

completing the square terms for the last term of (35), one may write (35) as

$$
\begin{aligned}
\dot{V}(t) \leq & -\lambda_{\text {min }}(\mathrm{A})\|e\|^{2}-\left(\lambda_{\text {min }}(\mathrm{B})-\frac{1}{2}\right)\left\|s_{1}\right\|^{2} \\
& -\lambda_{\text {min }}(\mathrm{C})\|z\|^{2}-\left(\lambda_{\text {min }}(\mathrm{D})-\frac{1}{2}\right)\left\|s_{2}\right\|^{2} \\
& -\mu_{\sigma}\left(1-\frac{1}{2 \kappa^{2}}\right)\|\tilde{W}\|_{\mathrm{F}}^{2}+\frac{1}{2} \mu_{\sigma} \kappa^{2}\|W\|_{\mathrm{F}}^{2}+\frac{1}{2}\|\varepsilon\|^{2}
\end{aligned}
$$

By providing the conditions $\lambda_{\min }(B)>\frac{1}{2}, \lambda_{\min }(D)>\frac{1}{2}$.

the Lyapunov function (29) can be stated as:

$$
\dot{V}\left(e, s_{1}, z, s_{2}, \tilde{W}\right) \leq-\lambda_{1} V\left(e, s_{1}, z, s_{2}, \tilde{W}\right)+\beta_{1}
$$

where

$$
\begin{aligned}
& \lambda_{1}=\min \left\{\frac{2 \lambda_{\text {min }}(\mathrm{A})}{\lambda_{\text {max }}\left(\mathrm{K}_{2}\right)}, \frac{2 \lambda_{\text {min }}(\mathrm{B})}{\lambda_{\text {max }}\left(\mathrm{M}_{2}\right)}, \frac{2 \lambda_{\text {min }}(\mathrm{C})}{\lambda_{\text {max }}\left(\mathrm{K}_{2}\right)}, \frac{2 \lambda_{\text {min }}(\mathrm{D})}{\lambda_{\text {max }}\left(\mathrm{M}_{2}\right)}\right\} \\
& \beta_{1}=\frac{1}{2} \mu_{\sigma} \kappa^{2}\|W\|_{\mathrm{F}}^{2}+\frac{1}{2}\|\varepsilon\|^{2}
\end{aligned}
$$

After solving the differential inequality (37), we have

$$
V(t) \leq V(0) e^{-\lambda_{1} t}+\beta_{1} / \lambda_{1}\left(1-e^{-\lambda_{1} t}\right), \quad \forall t \in[0, \infty)
$$

This implies that $\left(e, s_{1}, z, s_{2}, \tilde{W}\right)$ are uniformly ultimately bounded and exponentially converge to a small ball containing the origin. 
Remark 2: In order to make (21) and (23) independent from velocity measurements, the term $s_{1}+s_{2}$ is substituted by $\hat{s}_{1}+\hat{s}_{2}:=\dot{\hat{y}}-\dot{y}_{d}+\Lambda e$. In fact, if the gains are set large enough, the approximation $s_{1}+s_{2}=\hat{s}_{1}+\hat{s}_{2}$ is satisfied. The interested reader is referred to the reference [23].

\section{Application of the output feedback controller to a car-like WMR based on look-ahead control method}

Consider a car-like WMR which is moving on a horizontal plane as shown in Fig. 2. The robot has two fixed motorized wheels in the rear and two passive steering wheels in the front. Consider the following definition: $P_{C}=\left(x_{C}, y_{C}\right)$ : centre of mass of the robot, $P_{\mathbf{0}}=\left(x_{O}, y_{O}\right)$ : origin of the local coordinate frame, $P_{L}=\left(x_{L}, y_{L}\right)$ :virtual reference point, $q=\left[x_{O}, y_{O}, \theta, \varphi\right]^{T}:$ generalized coordinates vector, $l_{b}$ :denotes the distance between the front and rear wheels, $v(t)=\left[v_{1}(t), v_{2}(t)\right]^{T}$ : pseudo-velocities vector, $v_{1}$ : steering velocities, $v_{2}$ : linear velocities, two velocity constraints are obtained as follows:

$\left\{\begin{array}{c}-\dot{y}_{O} \cos (\theta+\varphi)+\dot{x}_{O} \sin (\theta+\varphi)-l_{b} \dot{\theta} \cos \varphi=0, \\ -\dot{y}_{O} \cos \theta+\dot{x}_{O} \sin \theta=0 .\end{array}\right.$

According to Lagrangian mechanics, the following matrices are adopted for the WMR models which are given by (1) and (3) [27]:

$M(\mathfrak{q})=\left[\begin{array}{cccc}m_{c} & 0 & -m_{c} l_{c} \sin \theta & 0 \\ 0 & m_{c} & m_{c} l_{c} \cos \theta & 0 \\ -m_{c} l_{c} \sin \theta & m_{c} l_{c} \cos \theta & I_{c}+I_{f} & I_{f} \\ 0 & 0 & I_{f} & I_{f}\end{array}\right], S(\boldsymbol{q})=\left[\begin{array}{cc}\cos \theta & 0 \\ \sin \theta & 0 \\ 1 / l_{b} \tan \varphi & 0 \\ 0 & 1\end{array}\right]$, $C(q, \dot{q}) \dot{q}=\left[\begin{array}{c}-m_{c} l_{c} \dot{\theta}^{2} \cos \theta \\ -m_{c} l_{c} \dot{\theta}^{2} \sin \theta \\ 0 \\ 0\end{array}\right], B(\boldsymbol{q})=\left[\begin{array}{cc}0 & \cos \theta \\ 0 & \sin \theta \\ 0 & l_{b} \sin \varphi \cos \varphi \\ 1 & 0\end{array}\right], X_{1}=\left[\begin{array}{cc}1 & 0 \\ 0 & 1 / r\end{array}\right]$

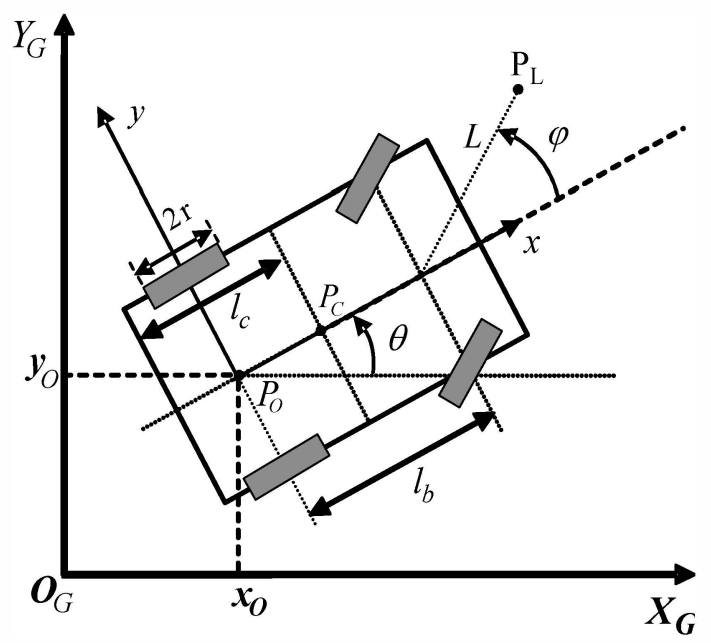

Fig. 2. Planar configuration of a car-like wheeled mobile robot.
The following output variables are chosen to track a desired trajectory based on look-ahead control method:

$$
y=h(q)=\left[\begin{array}{c}
x_{O}+l_{b} \cos \theta+L \cos (\theta+\varphi) \\
y_{O}+l_{b} \sin \theta+L \sin (\theta+\varphi)
\end{array}\right]
$$

\section{Simulation RESUlts}

In order to truthfully simulate a real world WMR system, the actuators inductances are not ignored in the simulation of actuators dynamics. This helps us evaluate the proposed controller in a more realistic situation. The WMR parameters are chosen as follows: $l_{c}=0.55 \mathrm{~m}, l_{b}=1.2 \mathrm{~m}, m_{c}=30 \mathrm{Kg}$, $I_{c}=5 \mathrm{Kgm}^{2}, \quad I_{f}=10 \mathrm{Kgm}^{2}, \quad n=48, \quad K_{t}=0.2 \mathrm{oz}-\mathrm{in} / \mathrm{A}$, $R_{a}=5 \Omega, L_{a}=0.1 \Omega \mathrm{s}, K_{b}=0.02 \mathrm{~V} / \mathrm{rad} / \mathrm{s}$.

It is assumed that the WMR is equipped with a relatively accurate localization system. However, Gaussian random noise with mean 0 and standard deviation $0.01 \mathrm{rad} / \mathrm{s}$ and 0.1 $\mathrm{cm} / \mathrm{s}$ is added to the steering and linear velocities of the robot, respectively, to simulate localization accumulative errors. The controller parameters are set to $\Lambda=\operatorname{diag}(1,0.5), k_{d}=30$, $K_{1}=50 I_{2 \times 2}, \quad K_{2}=60 I_{2 \times 2}, \quad \Gamma=50, \quad \sigma=0.02 \quad$ and $L=0.1 \mathrm{~m}$.

The following arbitrary desired trajectory is selected to evaluate the controller:

$$
y_{r}=\left[x_{g}+R \cos \left(\omega_{r} t\right), y_{g}+R \sin \left(\omega_{r} t\right)\right]^{T}
$$

The steering angle $\varphi$ is saturated such that $|\varphi(t)| \leq \pi / 3$ to avoid the matrix singularity in the proposed controller. The control signals are saturated within $\left|u_{a}\right| \leq 24 \mathrm{~V}$ to simulate the actuators saturation.

In order to evaluate the tracking performance and robustness of the proposed control system in a realistic situation, we consider the following non-parametric uncertainties on the robot: (i) it is supposed that the WMR chassis is subjected to varying loads. (ii) the friction and unmodeled dynamics are considered as $\tau_{d 1}=0.8 v+0.5 \operatorname{sgn}(v)+[5 \sin (0.05 t), 5 \sin (0.05 t)]^{T} \quad$ where the first term denotes the viscous friction, the second term is the coulomb friction and third tem denotes unmodeled dynamics such as passive wheels.

Fig. 3 shows the desired trajectory and the WMR trajectory which is generated by the proposed controller under these conditions. The tracking errors are illustrated by Fig. 4 These figures show that the proposed controller presents a satisfactory tracking performance and robustness which leads to a successful navigation for the car-like WMR which is subjected to parametric and non-parametric uncertainties. The control signals are also shown by the Fig. 5 .

\section{CONCLUSION}

The adaptive trajectory tracking control problems using position measurement for uncertain nonholonomic car-like driven wheeled mobile robots using RBF neural network have been proposed and solved. The developed control scheme is 
based on an adaptive NN system and an observer system. From the simulation results, it is concluded that the proposed design achieves the desired results. Thus, future work is the design of an output feedback controller for a car-like driven uncertain nonholonomic WMR considering actuators dynamics.

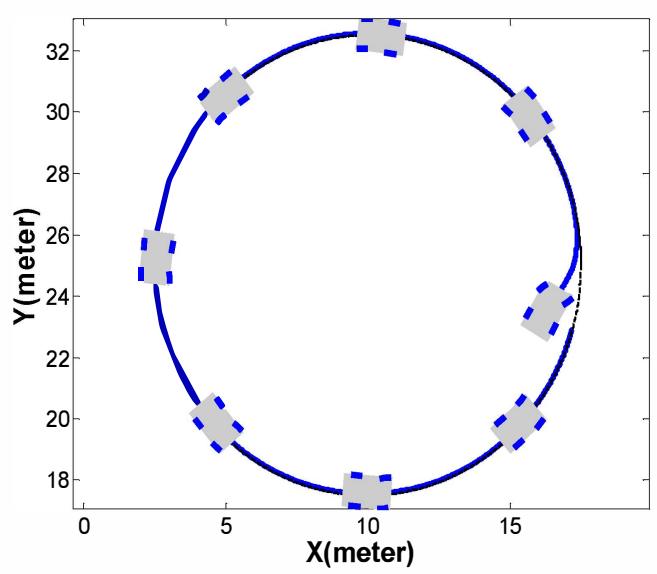

Fig. 3. The desired trajectory and WMR trajectory .
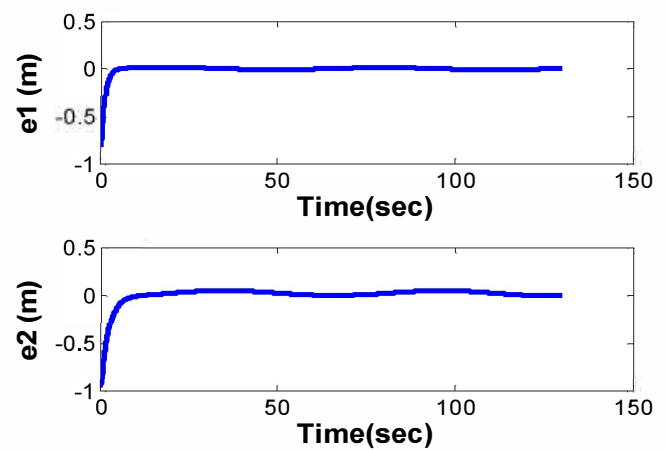

Fig. 4. Output tracking errors.
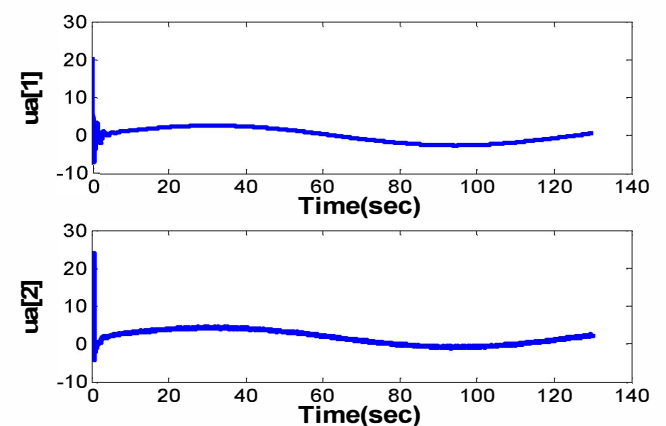

Fig. 5. Generated control signals

\section{REFERENCES}

[1] W. E. Dixon, M. S. de Queiroz, D. M. Dawson, T. J. Flynn, "Adaptive Tracking and Regulation of a Wheeled Mobile Robot with Controller/Update Law Modularity," IEEE Transactions of Control Systems Techonology, vol. 12 , no. 1, pp. 138-147, 2004.

[2] R. W. Brockett, "Asymptotic stability and feedback stabilization," in Differential Geometric Control Theory, R. W. Brockett, R.S. Milman, H. J. Sussman Eds., pp. 181-191, Boston, 1983.

[3] A. P. Aguiar, J. P. Hespanha, "Trajectory-Tracking and PathFollowing of Underactuated Autonomous Vehicles With Parametric Modeling Uncertainty," IEEE Tranactions on Automatic Control, vol. 52, no. 8, pp. 1362-1379, 2007.
[4] G. Campion, B. d'Andrea-Novel, G. Bastin, "Modeling and State Feedback Control of Nonholonomic Mechanical Systems," Proceedings of the 30th Conference on Decision and Control IEEE, pp. 11841189, England 1991

[5] C. Samson, K. Ait-Abderrahim, "Feedback control of a nonholonomicwheeled cart in Cartesian space," Proc. IEEE Int. Conf. Robotics and Automation, pp. 1136-1141, 1991.

[6] N. Sarkar, X. Yun, V. Kumar, "Control of mechanical systems with rolling constraint: Application to dynamic control of mobile robots," International Journal of Robotic Research, vol.13, no.1, pp. 55-69, 1994.

[7] C. de Wit, H. Khennouf, "Quasi-Continuous Stabilizing Controllers for Nonholonomic Systems: Design and Robustness Considerations," Proceedings of the European Control Conference, pp.2630-2635, 1995.

[8] Y. Kanayama, Y. Kimura, F. Miyazaki, T. Nogushi, "A stable tracking control method for an autonomous mobile robot," Proceedings of IEEE International conference on robotics and automation, pp. 384-389, 1990.

[9] Z. Jiang, H. Nijmeijer, " Tracking Control of Mobile Robots: A case study in Backstepping," Automatica, vol. 33, no. 7,pp. 1393-1399.

[10] R. Fierro, F. L. Lewis "Control of a nonholonomic mobile robot: backstepping kinematics into dynamics," Proc. 34th Conf. Decision and Control, pp. 3805-3810, LA, USA, December 1995.

[11] Kh. Shojaei, A. Mohammad Shahri, "Output feedback tracking control of uncertainnon-holonomic wheeled mobile robots: a dynamic surface control approach," Journal of Control Theory \& Applications IET, vol. 6, pp. 1751-8644, 2012.

[12] Kh. Shojaei, A. Mohammad Shahri, A. Tarakameh, B. Tabibian, "Adaptive trajectory tracking control of a differential drive wheeled mobile robot," Robotica, vol. 29, pp. 391-402, 2010.

[13] H.C. Cho, M.S. Fadali, K.S. Lee, N.H. Kim, "Adaptive position and trajectory control of autonomous mobile robot systems with random friction," IET Control Theory Appl, vol. 4, pp. 2733-2742, 2010.

[14] B.S. Park, S.J. Yoo, J.B. Park, Y.H . Choi, "A simple adaptive control approach for trajectory tracking of electrically driven nonholonomic mobile robots," IEEE Trans. Control Syst, vol. 18, pp. 1199-1206, 2010.

[15] D. Chwa, "Tracking control of differential-drive wheeled mobile robots using a backstepping-like feedback linearization," IEEE Trans. Syst. Man Cybern, vol. 40, pp. 1285-1295, 2010.

[16] K. D. Do, J. Pan, "Global output-feedback path tracking of unicycletype mobile robots," Robot. Comput. Integr., vol. 22, pp. 166-179. 2006

[17] H. Talebi Abatari, A. Dehghani Tafti " Using a Fuzzy PID Controller for the Path Following of a Car-like Mobile Robot,"Robotics and Mechatronics, pp. $189-193,2013$.

[18] A. D. Luca, G. Oriolo, C. Samso, "Feedback control of a nonholonomic car-like robot," in Robot Motion Planning and Control, ser. Lecture Notes in Control and Information Sciences, J. Laumond, Ed. Springer Berlin, vol. 229, pp. 171-253, 1998.

[19] C. B. Low, D. Wang, "GPS-Based Path Following Control for a CarLike Wheeled Mobile Robot With Skidding and Slipping," IEEEControl Systems Technology, vol. 16, pp. 1063-6536, March 2008.

[20] F. Hamerlain, “ Trajectory tracking control of a car-like mobile robot in presence of sliding, " IEEE Conf. on Control, pp. 502-507, 2012.

[21] M. Egerstedt, A. Stotsky, X. Hu, "control of a car-like robot using a dynamic model," IEEE Robotics and Automation, vol. 4, pp. 3273 3278,1998

[22] A. Stotsky, X. Hu , "Adaptive/variable structure control of carlike mobile robot in 4wheel dynamical model framework", 34-th IEEE Conf. on Decision and Control, Tampa FL,vol.3,pp.3111- 3116, 1998.

[23] M. A. Arteaga, R. Kelly, "Robot Control Without Velocity Measurements: New Theory and Experimental Results," IEEE Transaction on Robotics and Automation, vol. 20, pp. 297-308, 2004.

[24] R. Fierro, F. L. Lewis, " Control of Nonholonomic mobile robots: backstepping kinematics into dynamics," In Proc. 34-th IEEE Conf. on Decision and Control, New Orleans, vol. 4, pp. 3805-3810, 1998.

[25] F. L. Lewis, C. T. Abdallah, D. M. Dawson, Control of Robot Manipulators, MacMillan, New York, 1993.

[26] X. Yun, Y. Yamamoto, "Stability analysis of the internal dynamics of a wheeled mobile robot," J. Robot. Sys., vol. 14, no. 10,pp.697-709, 1997.

[27] C.S. Chiu, K.Y. Lian "Hybrid Fuzzy Model-Based Control of Nonnholonomic Systems: A Unified viewpoint," IEEE Transaction on Fuzzy Systems, vol. 16, no. 1, pp. 85-96, 2008. 